\title{
Heavy quark perturbative QCD fragmentation functions in the presence of hadron mass
}

\author{
S. Mohammad Moosavi Nejad ${ }^{1,2}$, Aida Armat ${ }^{1}$ \\ 1 Faculty of Physics, Yazd University, P.O. Box 89195-741, Yazd, Iran \\ 2 School of Particles and Accelerators, Institute for Research in Fundamental \\ Sciences (IPM), P.O.Box 19395-5531, Tehran, Iran
}

Received: date / Revised version: date

\begin{abstract}
The dominant mechanism to produce hadronic bound states with large transverse momentum is fragmentation, that is the splitting of a high energy parton into a hadronic state and other partons. We review the present schemes to calculate the heavy quark fragmentation functions (FFs) and drive an exact analytical expression of $\mathrm{FF}$ which includes most of the kinematical and dynamical properties of the process. Using the perturbative QCD, we calculate the FF for $c$-quark to split into $S$-wave $D^{+}$meson to leading order in the QCD coupling constant. Our result is compared with the current well-known phenomenological models which are obtained through a global fit to $e^{+} e^{-}$data from SLAC SLC and CERN LEP1 and we also compare the FF with experimental data form BELLE and CLEO. Specifically, we study the effect of outgoing meson mass on the pQCD FF. Meson masses are responsible for the low- $z$ threshold, where $z$ is the scaled energy variable.
\end{abstract}

\section{Introduction}

Hadron production processes are important in investigating properties of quarks in heavy ion collisions and in finding the origin of the nucleon spin in lepton-nucleon scattering processes and polarized proton-proton collisions. In order to calculate the hadron production cross section, the fragmentation functions (FFs) are the key quantities and they must be known in advance. The FFs describe hadron production probabilities from the initial partons and they cannot be precisely calculated by theoretical approaches at this stage. The FFs are related to the low energy part of the hadron production processes and they form the nonperturbative aspect of QCD. The FFs are universal and their importance is for model independent predictions of the cross sections at the Large Hadron Collider (LHC) in which a hadron is detected in the outgoing productions as a colorless bound state. They can also be used to find the internal structure of the exotic hadrons using the differences between the disfavored and favored FFs [1. The QCD improved parton model provides a great theoretical frame to extract these functions. However, once they are given at the initial fragmentation scale $\mu_{0}$, their $\mu$ evolution is determined by the Dokshitzer-Gribov-LipatovAlteralli-Parisi (DGLAP) renormalization group equations 2 which are very similar to those for parton distribution functions (PDFs). The universality of the initial condition of the FFs, first was suggested in [3] in the framework of $e^{-} e^{+}$annihilation and afterward was proved in a more general way in Ref. [4].

There are two main approaches to evaluate the FFs. In the first approach, which is frequently used to obtain the FFs, these functions are extracted from experimental data analyses instead of theoretical calculations. In this scheme, which is normally called the phenomenological approach, the FFs are mainly determined by hadron production data of $e^{-} e^{+}$annihilation, lepton-hadron and hadron-hadron scattering processes by working either in $x$-space [5, 6, 7, 8 or in Mellin-N space 9, 10. This situation is very similar to the determination of the PDFs. In this approach, according to the Collin's factorization theorem [11 the cross section of hadron production in the $e^{-} e^{+}$annihilation is described by the convolution of partonic hard-scattering cross sections $\left(e^{-} e^{+} \rightarrow q \bar{q}\right)$ which are calculable perturbatively and a realistic fragmentation function describing the transition of a parton into an outgoing hadron. In this scheme, the FFs involve parameters to be fixed by fitting the experimental data. Various phenomenological models like Peterson model [12, Lund model [13, Cascade model 14 and etc, have been developed to describe the FFs.

The second approach is based on this fact that the FFs for mesons containing a heavy quark can be computed theoretically using perturbative QCD (pQCD) [15, 16, 17, 18, [19. The first theoretical attempt to explain the procedure of hadron production by a heavy quark was made by Bjorken [20] by using a naive quark-parton model (QPM). He deduced that the inclusive distribution of heavy hadron should peak almost at $z=1$, where $z$ refers to the scaled energy variable. This property is important for heavy quarks for which the peak of heavy quark fragmentation function occurs closer to $z=1$. In continuation, Peterson 12 pre- 
sented the popular form of $\mathrm{FF}$ which manifestly behaves as $(1-z)^{2}$ at large $z$ values, using a quantum mechanical parton model. The pQCD scheme was followed by Suzuki 21], Amiri and Ji [22. While in this scheme Suzuki calculates the heavy FFs using a diagram similar to that in Fig. 1].

Here, we focus on heavy quark FFs and drive an exact analytical form of FF, using the Suzuki's approach which embeds most of the kinematical and dynamical properties of the process. Our results are compared both with one of the well-known phenomenological models and with the experimental data and they are found in good agreement. Furthermore, we impose finite meson mass effect on the perturbative QCD FF. This modifies the relations between partonic and hadronic variables and reduces the available phase space and is responsible for the low-z threshold. In Ref. [8], the effect of finite hadron mass on the nonperturbative fragmentation function is studied and it is shown that the inclusion of finite mass effect tends to improve the overall description of the data. Specifically, it is shown that hadron mass effect turned out to be more important than quark mass effects. Although this additional effect is not expected to be truly sizable numerically, its study is nevertheless necessary in order to fully exploit the enormous statistics of the LHC data to be taken in the long run for a high-precision determination of the topquark properties.

This paper is organized as follows. In Sec. 2] we explain the phenomenological approach to calculate the FFs by introducing a well-known model. In Sec. 3, the theoretical scheme to calculate the FFs is introduced in detail. We then discuss the use of the pQCD fragmentation functions as a phenomenological model for the fragmentation of the charm quark into the heavy-light mesons $D^{0}$ and $D^{+}$. In Sec. 4 we study, for the first time, the effect of meson mass on the perturbative QCD FFs and we present the numerical results and in Sec. 5] our conclusions are summarized.

\section{Determination of fragmentation functions: Phenomenological scheme}

One of the most current approaches to determine the FFs is the method based on data analyzing. The FFs are studied in hadron-hadron, lepton-hadron deep inelastic scattering (DIS) and electron-positron annihilation. Among all, the FFs are mainly determined by hadron production data of $e^{-} e^{+}$annihilation. The perturbative QCD framework is used to study single-inclusive hadron production in $e^{-} e^{+}$annihilation, where the factorization theorem is an important tool to study this process. According to the factorization theorem of the QCD improved parton model [11, in the high energy scattering the cross section can be expressed in terms of the partonic hard scattering cross sections and the non-perturbative FFs $D_{i}^{H}\left(z, Q^{2}\right)$ in which the last one is related to the low energy components of process, i.e.

$$
\frac{1}{\sigma_{t o t}} \frac{d}{d z} \sigma\left(e^{+} e^{-} \rightarrow H X\right)=\sum_{i} C_{i}\left(z, \alpha_{s}\right) \otimes D_{i}^{H}\left(z, Q^{2}\right),
$$

where, the function $D_{i}^{H}\left(z, Q^{2}\right)$ indicates the probability to find the hadron $H$ from a parton $i(=g, u, d, s, \cdots)$ with the energy fraction $z$ and $C_{i}\left(z, \alpha_{s}\right)$ is the Wilson coefficient function based on the partonic cross section $e^{+} e^{-} \rightarrow$ $q \bar{q}$ which is calculated in the perturbative QCD [7, 23], and the convolution integral is defined as $f(z) \otimes g(z)=$ $\int_{z}^{1} d y / y f(y) g(z / y)$. In the equation above, $X$ stands for the unobserved jets and $\sigma_{\text {tot }}$ is the total hadronic cross section 8, and $Q^{2}$ is the squared center-of-mass energy $s=Q^{2}$. The variable $z$ stands for the fragmentation parameter and is defined by the energy fraction

$$
z=\frac{2 E_{H}}{\sqrt{s}}
$$

where $E_{H}$ is the energy of detected hadron. In fact, the fragmentation parameter refers to the energy fraction of process which is taken away by the outgoing hadron $H$.

In the phenomenological approach, the FFs are parameterized in a convenient functional form at the initial scale $\mu_{0}^{2}$ in each order, i.e. LO and NLO. The initial scale $\mu_{0}^{2}$ is different for partons and the initial FFs are evolved to the experimental $\mu^{2}$ points by the DGLAP evolution equations 2. The FFs are parameterized in terms of a number of free parameters which are determined by an $\chi^{2}$ analysis of the $e^{+} e^{-} \rightarrow H+X$ data at the scale $\mu^{2}=s$. Due to the energy conservation, there is the following constraint for the parameters

$$
\sum_{H} \int_{0}^{1} d z z D_{i}^{H}\left(z, Q^{2}\right)=1
$$

This constraint is known as the energy sum rule, which means that each parton will fragment into some hadrons $H$.

In Ref. 24, authors calculated the $b \rightarrow B$ FFs based on the Peterson and power ansaetze obtained through a global fit to $e^{+} e^{-}$data from CERN LEP1 and SLAC SLC. In Ref. 8], authors reported the FFs for $D^{0}, D^{+}$ and $D^{\star+}$ mesons by fitting the experimental data from the BELLE, CLEO, ALEPH, and OPAL collaborations in the modified minimal-subtraction $(\overline{M S})$ factorization scheme. They have parameterized the $z$ distributions of the $c$ and $b$ quark FFs at their starting scales $\mu_{0}=m_{c}$ and $m_{b}$, respectively, as suggested by Bowler [25], as

$$
D_{q}^{H_{c}}\left(z, \mu_{0}\right)=N z^{-\left(1+\gamma^{2}\right)}(1-z)^{a} e^{-\gamma^{2} / z},
$$

with three free parameters. As they claimed, this parametrization yields the best fit to the BELLE data [26] in a comparative analysis using the Monte Carlo event generator JETSET/PYTHIA. The values of fit parameters for the $D^{+}(c \bar{d})$ meson are obtained from the BELLE/CLEO, 
Table 1. Values of fit parameters for $c \rightarrow D^{+} \mathrm{FF}$ at the starting scale $\mu_{0}=m_{c}=1.5 \mathrm{GeV}$ obtained from the Belle/CLEO, OPAL, and global fits in the ZM approach together with the values of $\overline{\chi^{2}}$ achieved.

\begin{tabular}{ccccc}
\hline & $N$ & $a$ & $\gamma$ & $\overline{\chi^{2}}$ \\
\hline Belle/CLEO-ZM & $7.30 \times 10^{5}$ & 1.12 & 3.43 & 1.37 \\
OPAL-ZM & $2.62 \times 10^{4}$ & 1.48 & 2.91 & 0.507 \\
Global-ZM & $7.31 \times 10^{5}$ & 1.13 & 3.43 & 2.21
\end{tabular}

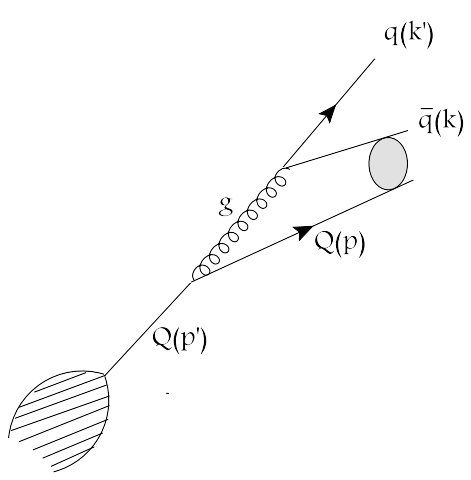

Fig. 1. Formation of a heavy meson. A heavy quark $Q$ forms a bound state $Q \bar{q}$ with a light antiquark produced through a single vector gluon.

OPAL, and the global fits using the massless scheme or zero-mass variable-flavor-number (ZM-VFN) scheme [27] where $m_{q}=0$ is put from the beginning and the non-zero values of the $c$ and $b$ quark masses only enter through the initial conditions of the non-perturbative FFs. The values of fit parameters together with the achieved values of $\overline{\chi^{2}}$ are reported in Table 1

\section{Heavy quark fragmentation functions: Perturbative QCD scheme}

As is pointed out in Refs. [16, 17, 18, the fragmentation function $D_{Q \rightarrow M}\left(z, \mu_{0}\right)$ for meson $M$ containing a heavy quark or a heavy antiquark $Q$ can be computed using the perturbative QCD. Here, the fragmentation parameter $z$ is the longitudinal momentum fraction of hadron relative to the fragmenting heavy quark, i.e.

$$
z=\frac{\left(E+p_{\|}\right)_{\text {hadron }}}{\left(E+p_{\|}\right)_{\text {beam }}} .
$$

In this work, using pQCD we apply the theoretical approach proposed by Suzuki 28, which is independent of data analyzing and is based on the convenient Feynman diagrams and the wave function of the heavy meson bound state. Therefore, at first, we briefly explain the Suzuki's approach to obtain the analytical FF of a heavy quark $Q$ into a heavy meson $M$ with a bound state $Q \bar{q}$. The main Feynman diagram for $Q \rightarrow M(Q \bar{q})+q$ in the order of $\alpha_{s}^{2}$ including the four-momenta is shown in Fig. 1 According to the Lepage-Brodsky's approach [29], by neglecting the relative motion of $Q$ and $\bar{q}$, we assume for simplicity that $Q$ and $\bar{q}$ are emitted collinearly with each other and they move along the $z$-axes. Following Ref. [28, we also adopt the infinite momentum frame where the fragmentation parameter $z$ (5) is reduced to another popular form as

$$
z=\frac{E_{\text {meson }}}{E_{\text {beam }}}
$$

We also set the relevant four-momenta in Fig. 1 as

$$
\begin{array}{ll}
p_{\mu}^{\prime}=\left[p_{0}^{\prime}, \boldsymbol{k}_{\perp}, p_{L}^{\prime}\right] & p_{\mu}=\left[p_{0}, \mathbf{0}, p_{L}\right] \\
k_{\mu}^{\prime}=\left[k_{0}^{\prime}, \boldsymbol{k}_{\perp}, k_{L}^{\prime}\right] & k_{\mu}=\left[k_{0}, \mathbf{0}, k_{L}\right],
\end{array}
$$

and the momentum of the produced meson is set as $\bar{p}_{\mu}=$ $\left[\bar{p}_{0}, \mathbf{0}, \bar{p}_{L}\right]$ where $\bar{p}_{L}=p_{L}+k_{L}$. We also may write the quark energies in terms of the initial heavy quark energy $p_{0}^{\prime}$ and the fragmentation parameter as

$$
p_{0}=x_{1} z p_{0}^{\prime}, \quad k_{0}=x_{2} z p_{0}^{\prime}, \quad k_{0}^{\prime}=(1-z) p_{0}^{\prime},
$$

where $x_{1}=p_{0} / \bar{p}_{0}$ and $x_{2}=k_{0} / \bar{p}_{0}$ are the meson energy fractions carried by the constituent quarks. As in Ref. [30, it is also assumed that the contribution of each constituent quark from the meson energy is proportional to its mass, i.e. $x_{1}=m_{Q} / M$ and $x_{2}=m_{\bar{q}} / M$ where $M=m_{Q}+m_{\bar{q}}$. Following Refs. 28,30, the fragmentation function may be defined as

$$
\begin{aligned}
D_{Q \rightarrow M}\left(z, \mu_{0}\right)=\frac{1}{\sigma} \frac{d \sigma}{d z} \\
\quad=\int d^{3} \boldsymbol{p} d^{3} \boldsymbol{k} d^{3} \boldsymbol{k}^{\prime} \overline{\left|T_{Q \rightarrow M}\right|^{2}} \delta^{3}\left(\boldsymbol{k}+\boldsymbol{p}+\boldsymbol{k}^{\prime}-\boldsymbol{p}^{\prime}\right),
\end{aligned}
$$

where the average probability amplitude squared $\overline{|T|^{2}}$ is obtained as $\sum_{s}|T|^{2} /\left(1+2 s_{Q}\right)$ in which the summation is going over the spins and colors and $s_{Q}$ is the initial heavy quark spin. The probability amplitude $T_{Q \rightarrow M}$ is expressed as the convolution of the hard scattering amplitude $T_{H}$, which can be computed perturbatively from quark-gluon subprocesses, and the process-independent distribution amplitude $\Phi_{M}$ which contains the bound state nonperturbative dynamic of outgoing meson, i.e.

$T_{Q \rightarrow M}=\int d x_{1} d x_{2} \delta\left(1-x_{1}-x_{2}\right) T_{H}\left(x_{i}, Q^{2}\right) \Phi_{M}\left(x_{i}, Q^{2}\right)$.

This scheme, introduced in 31,32, is used to absorb the soft behavior of the bound state into the hard scattering amplitude $T_{H}$. In (10), $\Phi_{M}$ is the probability amplitude to find the quarks which are collinear in the mesonic bound 
state up to the scale $Q$. In general, the probability amplitude $\Phi_{M}$ is related to the hadronic wave function $\Psi_{M}$ by

$$
\Phi_{M}\left(x_{i}, Q^{2}\right)=\int\left[d^{2} \boldsymbol{q}_{\perp i}\right] \Psi_{M}\left(x_{i}, \boldsymbol{q}_{\perp i}\right) \Theta\left(\boldsymbol{q}_{\perp i}^{2}<Q^{2}\right),
$$

where

$$
\left[d^{2} \boldsymbol{q}_{\perp \boldsymbol{i}}\right]=2(2 \pi)^{3} \delta\left[\sum_{j=1}^{2} \boldsymbol{q}_{\perp j}\right] \prod_{i=1}^{2} \frac{d^{2} \boldsymbol{q}_{\perp \boldsymbol{i}}}{2(2 \pi)^{3}} .
$$

The probability amplitude $\Phi_{M}$ represents the valence quark and antiquark wave function evaluated at quark impact separation $b_{\perp} \approx Q^{-1}$. Here, $\Theta(x)=\int_{-\infty}^{x} d t \delta(t)$ is the Heaviside step function and $\boldsymbol{q}_{\perp i}$ refers to the transverse momentum of constituent quarks. A typical simple mesonic wave function is

$$
\Psi_{M}\left(x_{i}, \boldsymbol{q}_{\perp i}\right)=\frac{\left(128 \pi^{3} b^{5} M\right)^{\frac{1}{2}}}{x_{1}^{2} x_{2}^{2}\left[M^{2}-\frac{m_{1}^{2}+\boldsymbol{q}_{\perp 1}{ }^{2}}{x_{1}}-\frac{m_{2}^{2}+\boldsymbol{q}_{\perp 2}{ }^{2}}{x_{2}}\right]^{2}},
$$

where $M$ is the meson mass and $b$ is the binding energy of the two body bound state. Both in the case $m_{1}=m_{2}$ and $m_{1}>m_{2}$, it can be shown that the above wave function is the solution of the Schrödinger equation with a Coulomb potential, which is the nonrelativistic limit of the Bethe-Salpeter equation with the QCD kernel [32].

Working in the infinite-momentum frame and by considering (11) and (13) we integrate over $\boldsymbol{q}_{\perp \boldsymbol{i}}\left(0 \leq \boldsymbol{q}_{\perp i}{ }^{2} \leq \infty\right)$ where $\boldsymbol{q}_{\perp \boldsymbol{i}}$ stands for $\boldsymbol{q}_{\perp \mathbf{1}}$ or $\boldsymbol{q}_{\perp \mathbf{2}}$. The integration yields an expression as

$\Phi_{M}\left(x_{i}, Q^{2}\right)=\frac{\left(128 \pi b^{5} M\right)^{\frac{1}{2}}}{16 \pi^{2}\left(x_{1}+x_{2}\right)\left(m_{1}^{2} x_{2}+m_{2}^{2} x_{1}-x_{1} x_{2} M^{2}\right)}$,

which grows rapidly at $x_{1}=1-x_{2}=m_{1} / M$ when $M$ is set to $m_{1}+m_{2}$ and therefore is estimated as a delta function [33. In conclusion for a S-wave pseudoscalar heavy meson $\left({ }^{1} S_{0}\right)$ with neglecting the Fermi motion, the probability amplitude at large $Q^{2}$ reads

$$
\Phi_{M} \approx \frac{f_{M}}{2 \sqrt{3}} \delta\left(x_{1}-\frac{m_{1}}{m_{1}+m_{2}}\right),
$$

where $f_{M}=\left(6 b^{3} / \pi M\right)^{\frac{1}{2}}$ refers to the decay constant for the meson. The delta-function form is convenient for our assumption where the constituent quarks inside the meson will fly together in parallel and they have no transverse momentum.

Considering Fig. 1, in which we make a simple approximation to form a meson by emitting only a single gluon, the hard scattering amplitude $T_{H}$ is expressed as

$T_{H}=\frac{2 \pi \alpha_{s} m_{q} M_{Q}}{\sqrt{2 p_{0} k_{0} k_{0}^{\prime} p_{0}^{\prime}}} \frac{C_{F}}{\left(k+k^{\prime}\right)^{2} D_{0}}\left\{\bar{u}(p) \gamma^{\mu} u\left(p^{\prime}\right) \bar{u}\left(k^{\prime}\right) \gamma_{\mu} v(k)\right\}$, where $D_{0}=p_{0}+k_{0}+k_{0}^{\prime}-p_{0}^{\prime}$ is the energy denominator, $C_{F}=\left(N_{c}^{2}-1\right) /\left(2 N_{c}\right)=4 / 3$ for $N_{c}=3$ quark colors, $\alpha_{s}$ is the strong coupling constant and $1 /\left(k+k^{\prime}\right)^{2}$ is due to the gluon propagator. Note that since the initial heavy quark is not on its mass shell, we have no energy conservation and thus we have performed the energy integration to reproduce the energy denominator $D_{0}$.

To obtain the $\mathrm{FF}$ for an unpolarized meson, considering (9) [16) and performing an average over the initial spin states and a sum over the final spin states we find

$$
\begin{aligned}
D_{Q \rightarrow M}\left(z, \mu_{0}\right)= & N \int \frac{d^{3} \boldsymbol{p} d^{3} \boldsymbol{k} d^{3} \boldsymbol{k}^{\prime} \delta^{3}\left(\boldsymbol{k}+\boldsymbol{p}+\boldsymbol{k}^{\prime}-\boldsymbol{p}^{\prime}\right)}{D_{0}^{2}\left(k+k^{\prime}\right)^{4} p_{0} k_{0} k_{0}^{\prime} p_{0}^{\prime}} \times \\
& {\left[2 m_{q}^{2} m_{Q}^{2}-m_{q}^{2}\left(p^{\prime} \cdot p\right)+m_{Q}^{2}\left(k \cdot k^{\prime}\right)\right.} \\
& \left.-(p \cdot k)\left(k^{\prime} \cdot p^{\prime}\right)-\left(k \cdot p^{\prime}\right)\left(p \cdot k^{\prime}\right)\right], \quad
\end{aligned}
$$

where $N$ is proportional to $\left(\pi C_{F} \alpha_{s} f_{M}\right)^{2}$ but it is related to the normalization condition $\int_{0}^{1} D_{Q}^{M}\left(z, \mu_{0}\right) d z=1$ [22, 28.

To do the phase space integrations we consider the following integral

$$
\begin{aligned}
& \int \frac{d^{3} \boldsymbol{p} \delta^{3}\left(\boldsymbol{k}+\boldsymbol{p}+\boldsymbol{k}^{\prime}-\boldsymbol{p}^{\prime}\right)}{p_{0} D_{0}^{2}} \\
& =\int \frac{d^{3} \boldsymbol{p} \delta^{3}\left(\boldsymbol{k}+\boldsymbol{p}+\boldsymbol{k}^{\prime}-\boldsymbol{p}^{\prime}\right)}{p_{0}\left(p_{0}+k_{0}+k_{0}^{\prime}-p_{0}^{\prime}\right)^{2}} \\
& =\int \frac{p_{0} d^{3} \boldsymbol{p} \delta^{3}\left(\boldsymbol{k}+\boldsymbol{p}+\boldsymbol{k}^{\prime}-\boldsymbol{p}^{\prime}\right)}{\left[p_{0}^{2}+p_{0}\left(k_{0}+k_{0}^{\prime}-p_{0}^{\prime}\right)\right]^{2}} \\
& =\frac{p_{0}}{\left[m_{Q}^{2}+\left(\boldsymbol{p}^{\prime}-\left(\boldsymbol{k}+\boldsymbol{k}^{\prime}\right)\right)^{2}-p_{0}\left(p_{0}^{\prime}-\left(k_{0}+k_{0}^{\prime}\right)\right)\right]^{2}} \\
& =\frac{p_{0}}{\left[\left(k+k^{\prime}\right)^{2}\right]^{2}},
\end{aligned}
$$

where considering (7) one has

$$
\left(k+k^{\prime}\right)^{2}=-m_{q} M J^{\frac{1}{4}}\left(z, k_{\perp}^{2}\right),
$$

with

$$
J\left(z, k_{\perp}^{2}\right)=\left[1-2 \frac{m_{q}}{M}-\frac{1}{z}-\frac{m_{q}^{2}+k_{\perp}^{2}}{M^{2}}\left(\frac{z}{1-z}\right)\right]^{4},
$$

and for the remaining integral we have

$$
\begin{aligned}
\int d^{3} \boldsymbol{k}^{\prime} f\left(z, \boldsymbol{k}_{\perp}^{2}\right) & =\int d k_{L}^{\prime} d^{2} k_{\perp} f\left(z, \boldsymbol{k}_{\perp}^{2}\right) \\
& \cong m_{q}^{2} k_{0}^{\prime} f\left(z,\left\langle k_{\perp}^{2}\right\rangle\right),
\end{aligned}
$$

where, for simplicity, we have replaced the transverse momentum integration by its average value $\left\langle k_{\perp}^{2}\right\rangle$, which is a free parameter and can be determined experimentally. Putting all in (9) we obtain the fragmentation function as

$D_{Q \rightarrow M}\left(z, \mu_{0}^{2}\right)=\frac{N}{z(1-z) J\left(z,\left\langle k_{\perp}^{2}\right\rangle\right)}\left\{R H \frac{m_{q}}{M^{2}}\right.$ 


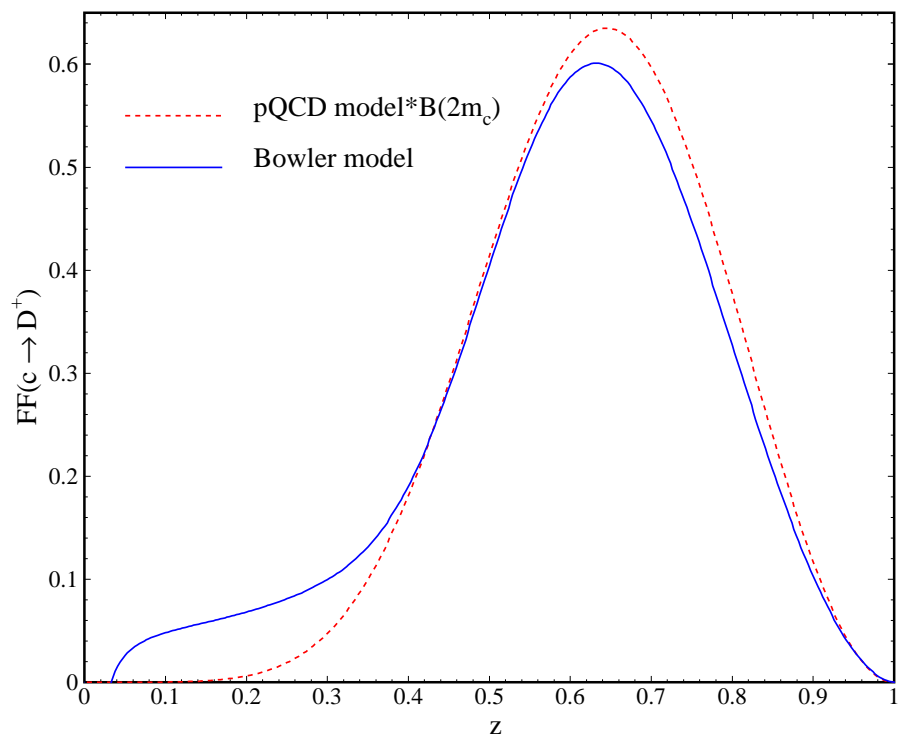

Fig. 2. $c \rightarrow D^{+} \mathrm{FF}$ at the initial scale $\mu_{0}=2 m_{c}$ as a function of $z$ in the pQCD approach (dashed line) and Bowler model (solid line).

$$
\begin{aligned}
& +\frac{z}{M}\left[\frac{T R}{1-z}-T H-m_{Q} m_{q} R\right]+ \\
& \left.2 m_{Q} z^{2}\left[T+m_{q}^{2}(1-z)\left(4-\frac{H}{z M m_{Q}}\right)\right]\right\}
\end{aligned}
$$

where,

$$
\begin{aligned}
& T\left(z,\left\langle k_{\perp}^{2}\right\rangle\right)=m_{q}^{2}+m_{Q}^{2}(1-z)^{2}+z^{2}\left\langle k_{\perp}^{2}\right\rangle, \\
& H\left(z,\left\langle k_{\perp}^{2}\right\rangle\right)=M^{2}+z^{2}\left(m_{Q}^{2}+\left\langle k_{\perp}^{2}\right\rangle\right), \\
& R\left(z,\left\langle k_{\perp}^{2}\right\rangle\right)=M^{2}(1-z)^{2}+z^{2}\left(m_{q}^{2}+\left\langle k_{\perp}^{2}\right\rangle\right) .
\end{aligned}
$$

In general, fragmentation functions $D_{Q \rightarrow M}\left(z, \mu^{2}\right)$ depend on both $z$ and factorization scale $\mu$. The scale $\mu$ is arbitrary, but in a high energy process where a jet is produced with transverse momentum $k_{\perp}$, large logarithms of $k_{\perp} / \mu$ in the parton cross section $C_{i}\left(z, \alpha_{s}\right)$ (1) can be avoided by choosing $\mu$ on the order of $k_{\perp}$. The function (22) should be regarded as a model for heavy quark FF at a initial scale $\mu$ of order $m_{Q}$. Here we set the initial scale to $\mu_{0}=2 m_{Q}$. For values of $\mu$ much larger than $\mu_{0}$, the obtained $\mathrm{FF}$ should be evolved from the scale $\mu_{0}=2 m_{Q}$ to the scale $\mu$ using the Altarelli-Parisi equation,

$\frac{d}{d \ln \mu^{2}} D_{Q \rightarrow M}\left(z, \mu^{2}\right)=\int_{z}^{1} \frac{d y}{y} P_{Q \rightarrow Q}\left(\frac{z}{y}, \mu\right) D_{Q \rightarrow M}\left(y, \mu^{2}\right)$,

where $P_{Q \rightarrow Q}$ is the appropriate splitting function,

$$
P_{Q \rightarrow Q}(x, \mu)=\frac{2 \alpha_{s}(\mu)}{3 \pi}\left(\frac{1+x^{2}}{1-x}\right)_{+} .
$$

As an example we consider the fragmentation of $c$-quark into the $D^{+}$-meson with the constituent quark structure
$\left|D^{+}>=\right| c \bar{d}>$, considering $m_{Q}=m_{c}=1.5 \mathrm{GeV}$ as was used in [8], $m_{q}=m_{d}=3 \mathrm{MeV}$ and $\left\langle k_{\perp}^{2}\right\rangle=m_{c}^{2}$ in (22). In Fig. 2 the behavior of $D^{+} \mathrm{FF}$ at the starting scale $\mu_{0}=$ $2 m_{c}$ is shown. Using the non-perturbative FF parameters from the second row of Table 1 and by evolving the FF to the scale $\mu=2 m_{c}$ we also compare our result with the Bowler model as a well-known phenomenological model [25. Since to obtain the constant $N$ (22) we have used the normalization condition, then to compare our result with the Bowler model, our theoretical result should be multiplied by the $c \rightarrow D^{+}$branching fraction $B\left(2 m_{c}\right)=0.235$ [8], which is defined as $B(\mu)=\int_{z_{\text {cut }}}^{1} d z D\left(z, \mu^{2}\right)$ where the cut $z_{\text {cut }}$ excludes the $z$ range in which the result is not valid. As Fig. 2 shows our result is in reliable consistency with the phenomenological model. Note that the function in the pQCD approach is determined in leading order whereas the Bowler function is extracted in NLO. Therefore, we may also think of other effects, such as gluon radiation and secondary fragmentation and so on, which can make a better agreement with the phenomenological model. In spite of the uncertainties mentioned, there is another theoretical uncertainty due to the freedom in the choice of scaling variable $z$ which will be discussed in next section.

The $z$ dependences of the FFs are not yet calculable at each desired scale. However, once they are given at some initial fragmentation scale $\mu_{0}$, their $\mu_{f}$ evolution is specified by the evolution equations (24). Therefore, having the initial FF (22),$D_{c \rightarrow D^{+}}(z, \mu)$ at larger values of $\mu$ can be obtained by solving DGLAP equations. To illustrate the effects of evolution, we evolve the FF at the energy scales $\mu=10.52 \mathrm{GeV}$ and $\mu=m_{Z}=91.2 \mathrm{GeV}$. These results are shown in Fig. 3. Since, in the measurement and the analysis of the data performed by the Belle and the CLEO Collaborations [26], the center of mass energy has been set to $\sqrt{s}=10.52 \mathrm{GeV}$, which is much close to the production threshold of $D$ mesons, we chose this value. The evolution causes the FF to decrease at large $z$ and to diverge at $z=0$.

Besides the $c \rightarrow D^{+} \mathrm{FF}$ itself, also its first moment is also of phenomenological interest and subject to experimental determination. It corresponds to the average fraction of energy that the $D^{+}$meson receives from the $c$ quark, i.e.

$$
\langle z\rangle(\mu)=\frac{1}{B(\mu)} \int_{z_{c u t}}^{1} d z z D\left(z, \mu^{2}\right)
$$

where $z_{\text {cut }}=0.1$. As is seen from Figs. 4 and 5 , there are no experimental data at $z<0$.1. It is interesting to compare our result obtained for the average energy fraction $\langle z\rangle\left(m_{c}\right)=0.73$ with the values quoted by BELLE, CLEO, ALEPH and OPAL which are listed in 88. There is good consistency between our result and the experimental results, however one must keep in mind that experimental results naturally include all orders and also contributions from gluon and light-quark fragmentation, while ours are evaluated from the $c \rightarrow D^{+} \mathrm{FF}$ at LO via (26).

In the remainder of this section, we also compare $z$ distributions of $D^{+}$and $D^{0}$ mesons from BELLE and CLEO 


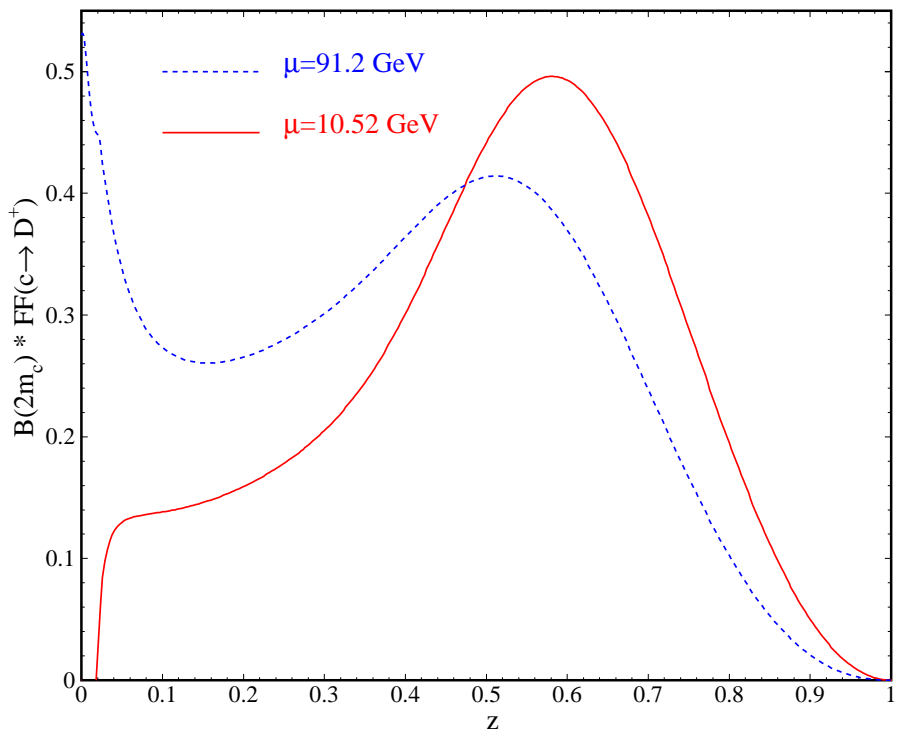

Fig. 3. The fragmentation function $D_{c}^{D^{+}}$as a function of $z$ for $\mu=10.52 \mathrm{GeV}$ (solid line) and $\mu=m_{z}$ (dashed line) normalized by the branching fraction $B\left(2 m_{c}\right)$.

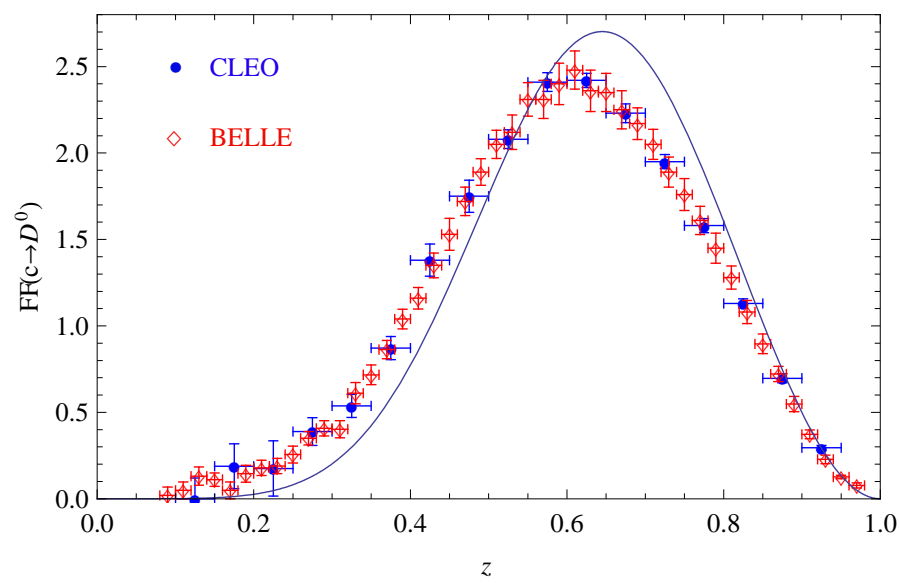

Fig. 4. Comparision of $\mathrm{pQCD} F \mathrm{~F}$ with data from BELLE and CLEO on $D^{0}$ production at the initial scale $\mu_{0}=2 m_{c}$. We set $\left\langle k_{\perp}^{2}\right\rangle=m_{c}^{2}$.

34 with our theoretical result. These are shown in Figs. 4 and 5 .

\section{Hadron Mass Effects on FFs}

In this section we find it instructive to concentrate on the massive kinematics of fragmentation, a topic with a very little attention paid to in the literature. Therefore, we show how to incorporate the effects of the hadron mass into the fragmentation function using a specific choice of scaling variable.

The FF depends on the fragmentation parameter $z$ (5) and there is some freedom in defining this parameter in

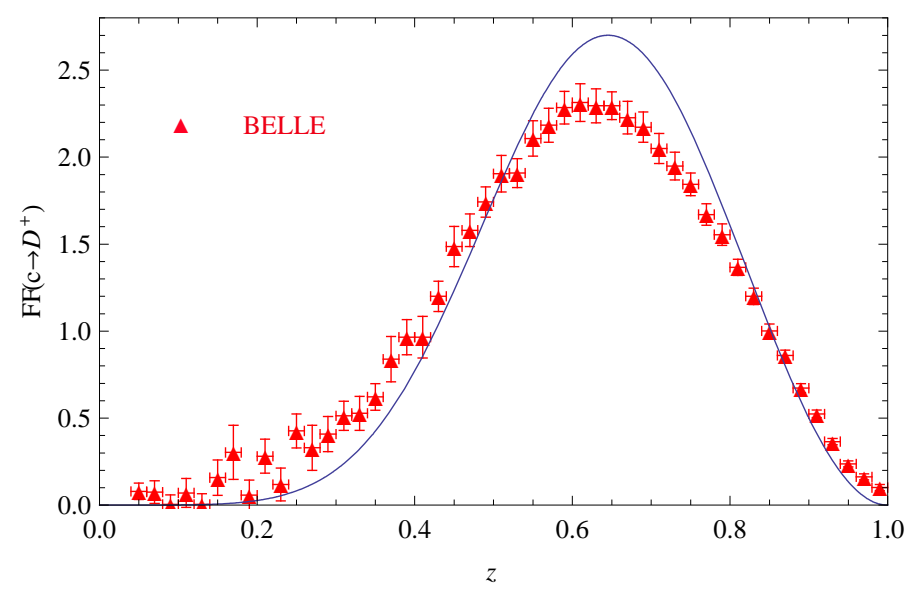

Fig. 5. As in figure 4 but comparing with $D^{+}$data from BELLE experiments at the initial scale $\mu_{0}=2 m_{c}$.

the presence of hadron mass. In general case the fourmomenta of the produced hadron $M$ and the mother parton $Q$ are related as $p_{M}=z p_{Q}$ that in the case of massless parton and hadron a custom choice is $E_{M}=z E_{Q}$ (see (6)) in which the scaling variable $z$ takes the values $0 \leq z \leq 1$, i.e. to retain just one of the four equations $p_{M}=z p_{Q}$. This simple relation is not suitable with the finite quark and/or hadron masses and needs to be generalized when a heavy quark and/or hadron is considered. In order to evaluate the theoretical uncertainty due to the freedom in the choice of fragmentation parameter in the presence of heavy quark and meson we use an approach given in Ref. 35, where authors took into account the finite mass corrections on the inclusive hadron production in $e^{+} e^{-}$ and hadron-hadron reactions. For this purpose it is helpful to work in light-cone coordinates, in which any fourvector $V$ is written in the form $V=\left(V^{+}, V^{-}, \boldsymbol{V}_{\boldsymbol{T}}\right)$ where $V^{ \pm}=\left(V_{0} \pm V_{3}\right) / \sqrt{2}$ and $\boldsymbol{V}_{\boldsymbol{T}}=\left(V_{1}, V_{2}\right)$. Considering (7) the momentum of the initial heavy quark takes the form

$$
p^{\prime}=\left[\frac{p_{0}^{\prime}+p_{L}^{\prime}}{\sqrt{2}}, \frac{p_{0}^{\prime}-p_{L}^{\prime}}{\sqrt{2}}, \boldsymbol{k}_{\perp}\right],
$$

and for the massless meson for which $\bar{p}_{0}=\bar{p}_{L}$ the fourmomentum is expressed as

$$
\bar{p}=\left[\sqrt{2} \overline{p_{0}}, 0, \mathbf{0}\right] .
$$

In the presence of meson and/or quark masses, the lightcone scaling variable $\zeta=\bar{p}^{+} / p^{\prime+}$ seems more convenient than the fragmentation parameter $z=\bar{p}_{0} / p_{0}^{\prime}$ (6). However, in the absence of meson and quark masses, the two variables are identical. Therefore, to study the effects of hadron mass on FF we apply the parameter $\zeta$ which is invariant with respect to boosts along the three-axis. This axis is considered as the flight direction of outgoing meson. Taking mass $M$ for the meson so that $\bar{p}^{2}=M^{2}$, the four-momenta of the meson in the light-cone coordinates reads

$$
\bar{p}=\left[\zeta p^{\prime+}, \frac{M^{2}}{2 \zeta p^{\prime+}}, \mathbf{0}\right] .
$$




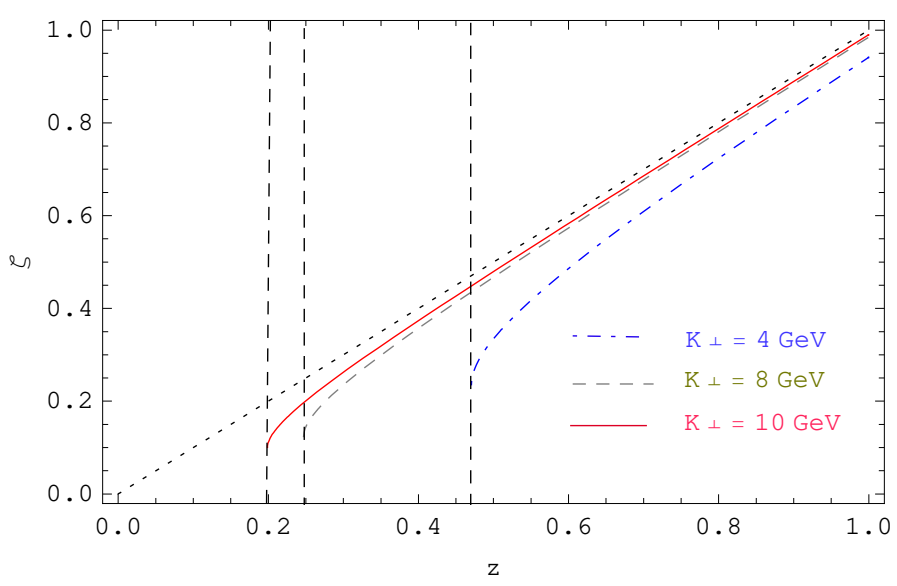

Fig. 6. The variations of $\zeta$ as a function of $z$ for $k_{\perp}=4 \mathrm{GeV}$, $k_{\perp}=8 \mathrm{GeV}$ and $k_{\perp}=10 \mathrm{GeV}$. Thresholds are also shown.

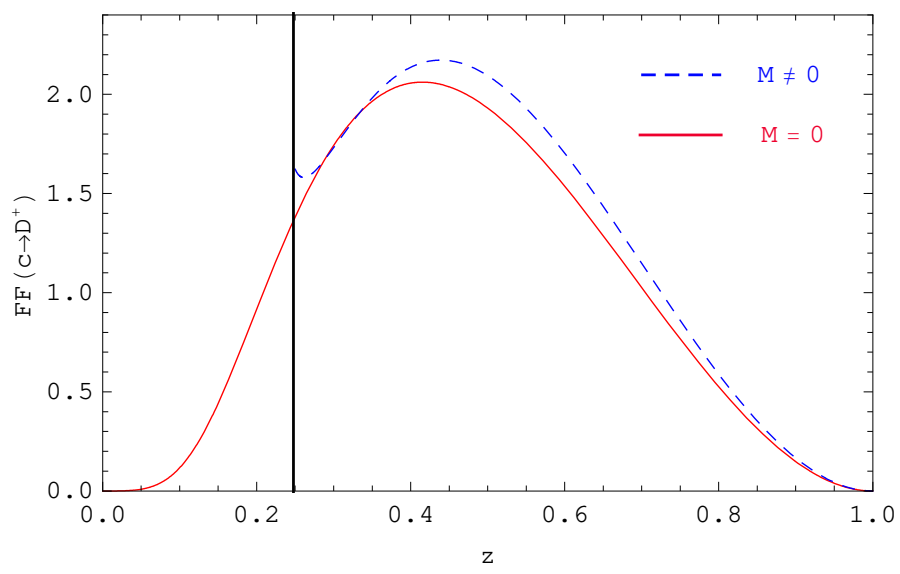

Fig. 7. $c \rightarrow D^{+} \mathrm{FF}$ as a function of $z$ when $M=0$ (solid line) and $M \neq 0$ (dashed line) taking $k_{\perp}=8 \mathrm{GeV}$. Threshold at $z=0.25$ is also shown

Comparison of (28) with (29) shows that the hadron mass effect is imposed by introducing a non-zero minus component into the hadron's momentum. From this result we obtain immediately the relation between the two scaling variables in the presence of hadron mass as

$$
z=\zeta\left(1+\frac{M^{2}}{\left(2 p_{0}^{\prime} \zeta\right)^{2}}\right)
$$

Note that these two variables are equal when $M \rightarrow 0$. Considering the four-momenta from (17) and at the fragmentation process with the sufficiently large transverse momentum, one can write $m_{Q}^{2} \approx p_{0}^{\prime 2}-k_{\perp}^{2}$. In Fig. 6, taking $m_{Q}=m_{c}=1.5 \mathrm{GeV}$ and $M=m_{D^{+}}=1.87 \mathrm{GeV} 36$ the variations of new scaling variable $\zeta$ as a function of $z$ is shown for different values of $k_{\perp}$. As it is seen the effect of meson mass is considerable when the transverse momentum $k_{\perp}$ decreases and this effect also creates a threshold for the FFs.

Now, to obtain the improved FF we go back to the defini- tion of fragmentation (9), i.e. $D_{Q \rightarrow M}(z)=1 / \sigma \cdot d \sigma(z) / d z$. As a generalization of the massless hadron case, we assume that the cross section which we have been calculating is $d \sigma(\zeta) / d \zeta$ which is related to the measured observable $d \sigma(z) / d z$ via

$$
\frac{d \sigma(z)}{d z}=\frac{d \zeta}{d z} \frac{d \sigma(\zeta(z))}{d \zeta}
$$

that is simplified as

$$
\frac{d \sigma(z)}{d z}=\frac{1}{1-\left(\frac{M}{2 p_{0}^{\prime} \zeta}\right)^{2}} \frac{d \sigma(\zeta(z))}{d \zeta}
$$

Here, using (30) our new scaling variable is expressed as

$$
\zeta=\frac{z}{2}\left\{1+\left(1-\frac{M^{2}}{z^{2}\left(m_{Q}^{2}+k_{\perp}^{2}\right)}\right)^{\frac{1}{2}}\right\},
$$

and now the observable quantity $D_{Q \rightarrow M}(z)$, reads

$$
D_{Q \rightarrow M}(z, \mu)=\frac{1}{1-\frac{M^{2}}{4 \zeta^{2}\left(m_{Q}^{2}+k_{\perp}^{2}\right)}} D_{Q \rightarrow M}(\zeta(z), \mu) .
$$

Note that $D_{Q \rightarrow M}(\zeta, \mu)$ is the FF obtained in (22) by substituting $z \rightarrow \zeta$ and the kinematically allowed $z$ ranges are now $M / \sqrt{m_{Q}^{2}+k_{\perp}^{2}}<z \leq 1$.

In Fig. 7 the behavior of $D^{+}$fragmentation function is shown for the massless and massive meson considering $m_{D^{+}}=1869.62 \mathrm{MeV}$. As is shown the effect of meson mass is increasing the size of FF at large values of $z$ and the peak position is shifted towards higher values of $z$ and it also creates a threshold at $z=0.25$.

\section{Conclusion}

We studied the heavy quark FFs in the current approaches. Using the perturbative QCD scheme we presented an analytical form for the FFs to produce S-wave heavy mesons to leading order in $\alpha_{s}$ which agrees with most kinematical and dynamical expectations. Our result describes not only the $z$ dependence of the fragmentation probabilities, but also their dependence on the transverse momentum of the meson relative to the produced jet. The perturbative QCD FF was compared with a well-known phenomenological model for the heavy quark fragmentation in the literature. Specifically, we compared the FFs for $D^{0}$ and $D^{+}$mesons with available $e^{-} e^{+}$annihilation data, from CLEO and BELLE 34 and we found good agreement. We also investigated, for the first time, finite meson mass corrections on the pQCD FFs and their theoretical uncertainty due to the freedom in the choice of the scaling variable. The advent of precise data from $D$ factories motivates the incorporation of hadron mass effect, which are then likely to be no longer negligible into the formalism. 


\section{References}

1. M. Hirai and S. Kumano, Prog. Theor. Phys. Suppl. 186 , 244 (2010).

2. V. N. Gribov and L. N. Lipatov, Sov. J. Nucl. Phys. 15, 438 (1972) [Yad. Fiz. 15, 781 (1972)]; G. Altarelli and G. Parisi, Nucl. Phys. B 126, 298 (1977); Yu. L. Dokshitzer, Sov. Phys. JETP 46, 641 (1977) [Zh. Eksp. Teor. Fiz. 73, 1216 (1977)].

3. B. Mele, P. Nason, Nucl. Phys. B 361 (1991) 626.

4. M. Cacciari and S. Catani, Nucl. Phys. B 617 (2001) 253.

5. Studies on fragmentation functions are listed in http://www.pv.infn.it/ radici/FFdatabase/

6. S. Albino, B. A. Kniehl and G. Kramer, Nucl. Phys. B 725 (2005) 181; B. A. Kniehl, G. Kramer and B. Potter, Nucl. Phys. B 597 (2001) 337.

7. B. A. Kniehl, G. Kramer and B. Potter, Nucl. Phys. B 582 (2000) 514.

8. T. Kneesch, B. A. Kniehl, G. Kramer and I. Schienbein, Nucl. Phys. B 799 (2008) 34.

9. P. Nason and C. Oleari, Nucl. Phys. B 565 (2000) 245.

10. M. Cacciari, P. Nason and C. Oleari, JHEP 0604 (2006) 006 .

11. J. C. Collins, Phys. Rev. D 58 (1998) 094002.

12. C. Peterson, D. Schlatter, I. Schmitt and P. M. Zerwas, Phys. Rev. D 27 (1983) 105.

13. B. Andersson, G. Gustafson, G. Ingelman and T. Sjostrand, Phys. Rept. 97 (1983) 31.

14. B. R. Webber, Nucl. Phys. B 238 (1984) 492.

15. J. P. Ma, Nucl. Phys. B 506 (1997) 329.

16. E. Braaten and T. C. Yuan, Phys. Rev. Lett. 71 (1993) 1673.

17. C. -H. Chang and Y. -Q. Chen, Phys. Lett. B 284 (1992) 127.

18. E. Braaten, K. -m. Cheung and T. C. Yuan, Phys. Rev. D 48 (1993) 4230.

19. D. M. Scott, Phys. Rev. D 18 (1978) 210.

20. J. D. Bjorken, Phys. Rev. D 17 (1978) 171.

21. M. Suzuki, Phys. Lett. B 71 (1977) 139.

22. F. Amiri and C. -R. Ji, Phys. Lett. B 195 (1987) 593.

23. P. Nason and B. R. Webber, Nucl. Phys. B 421 (1994) 473 [Erratum-ibid. B 480 (1996) 755].

24. B. A. Kniehl, G. Kramer, I. Schienbein and H. Spiesberger, Phys. Rev. D 84 (2011) 094026.

25. M. G. Bowler, Z. Phys. C 11 (1981) 169.

26. Belle Collaboration, R. Seuster, et al., Phys. Rev. D 73 , 032002 (2006); M. Artuso et al. [CLEO Collaboration], Phys. Rev. D 70 (2004) 112001.

27. J. Binnewies, B.A. Kniehl, and G. Kramer, Phys. Rev. D 58, 034016 (1998);

M. Cacciari and M. Greco, Nucl. Phys. B421, 530(1994).

28. M. Suzuki, Phys. Rev. D 33 (1986) 676.

29. G. P. Lepage and S. J. Brodsky, Phys. Rev. D 22 (1980) 2157.

30. M. A. Gomshi Nobary, J. Phys. G 20, 65 (1994).

31. A. D. Adamov and G. R. Goldstein, Phys. Rev. D 56 (1997) 7381

32. S. J. Brodsky and C. -R. Ji, Phys. Rev. Lett. 55 (1985) 2257.

33. F. Amiri, B. C. Harms and C. -R. Ji, Phys. Rev. D 32 (1985) 2982.

34. G. Corcella and G. Ferrera, JHEP 0712 (2007) 029.
35. S. Albino, B. A. Kniehl, G. Kramer and W. Ochs, Phys. Rev. D 73 (2006) 054020.

36. K. Nakamura et al. (Particle Data Group), J. Phys. G 37, 075021 (2010). 